ARTÍCULO DE INVESTIGACIÓN

\title{
Problemas de traducción, detección y descripción: un estudio longitudinal en la formación de traductores
}

Identifying and Describing Translation Problems: A Longitudinal Case-study in Translator Training Problemas de tradução, detecção e descrição: estudo longitudinal na formação de tradutoress

Ana Gregorio Cano* (https://orcid.org/0000-0001-7134-1150)

Department of Modern Languages, The University of Texas at Arlington, Arlington, Texas, U.S.A
Recibido: $08-08-17$

Revisado: $25-09-17$

Aceptado: $30-11-17$

Publicado: 18-12-17
RESUMEN. Esta investigación se enmarca dentro del área de la traducción e

Palabras clave: interpretación y, más específicamente, en el campo de la didáctica de la traducción. El objetivo general de la investigación de este estudio fue observar y analizar el impacto de la formación recibida en el seno de los estudios de Traducción en cinco universidades diferentes en España. Con el fin de alcanzar el objetivo de investigación se combinan los enfoques cuantitativos y cualitativos a través del diseño de un macro-estudio con más de 1.000 participantes y un estudio longitudinal de 37 estudiantes. Nuestra investigación se enfoca específicamente en el desarrollo de la competencia estratégica (CE) y, particularmente, la capacidad del estudiante para identificar y describir problemas de traducción. Además, en el caso del estudio longitudinal se formularon cuatro hipótesis para ser comprobadas o refutadas a partir de las respuestas de los estudiantes. En este artículo presentamos los resultados correspondientes a la hipótesis número 4, a saber: la riqueza del metalenguaje utilizado por los estudiantes de $\mathrm{Tl}$ evoluciona desde el principio hasta el final de su carrera. Los resultados del estudio longitudinal no demuestran patrones en el desarrollo de la CE, aunque el metalenguaje utilizado por los estudiantes experimenta cierta evolución. metalenguaje de la

Traducción, estudiante de traducción, problema de traducción, estudio longitudinal. 
ABSTRACT: This research is framed within the area of translation and interpreting studies and more specifically within the field of translation didactics. The general research objective of this study is to observe and analyze the repercussions of Translation undergraduate courses at five different universities in Spain. With empirical methods combining quantitative and qualitative analysis relying on a macro-study of more than 1,000 subjects and a longitudinal study of 37 students, the research focuses specifically on the development of the strategic competence (SC) and, particularly, the student's capacity to identify and describe translation problems. Furthermore, in the case of the longitudinal study, four hypotheses were tested against the students' answers. In this paper, we present the results of hypothesis number 4: whether the richness of the metalanguage used by the $\mathrm{Tl}$ students evolves from the beginning to the end of their study program. The longitudinal study results do not show any patterns for the development of the SC, although the metalanguage used by students does evolve.
Keywords:

Translation

metalanguage,

translation

student,

translation

problems,

longitudinal study.

RESUMO. Esta pesquisa faz parte da área de tradução e interpretação e, mais especificamente, no campo do ensino de tradução. 0 objetivo geral da pesquisa deste estudo foi observar e analisar o impacto do treinamento recebido em Estudos de Tradução em cinco universidades diferentes em Espanha. Para atingir o objetivo da pesquisa, as abordagens quantitativas e qualitativas são combinadas através do projeto de um macro-estudo com mais de 1000 participantes e um estudo longitudinal de 37 alunos. Nossa pesquisa se concentra especificamente no

Palavras-chave: tradução metalinguagem, estudante de tradução, problema de tradução, estudo longitudinal. desenvolvimento de competências estratégicas (CE) e, em particular, na capacidade do aluno de identificar e descrever problemas de tradução. Além disso, no caso do estudo longitudinal, quatro hipóteses foram formuladas para serem verificadas ou refutadas das respostas dos alunos. Neste artigo, apresentamos os resultados correspondentes à hipótese número 4, a saber: a riqueza de metalinguagem usada pelos estudantes de TI evolui desde o início até o final de sua carreira. Os resultados do estudo longitudinal não mostram padrões no desenvolvimento de CE, embora a metalinguagem utilizada pelos alunos sofra alguma evolução.

\section{INTRODUCCIÓN: DIDÁCTICA Y COMPETENCIA TRADUCTORA}

El estudio longitudinal que se presenta en estas páginas, así como los resultados del mismo, surgen en el marco de un proyecto I+D financiado por el Ministerio de Educación, Cultura y Deporte, titulado "Adquisición de la competencia cultural e intercultural (ACCI)", que tenía por objeto el análisis de la competencia traductora (CT). 
Nuestra investigación, por tanto, se enmarca en la didáctica de la traducción entendida como la disciplina que se encarga de la resolución de problemas relativos al proceso de enseñanza-aprendizaje de la traducción, de la elaboración de metodologías adecuadas para alcanzar dicho objetivo (aprendizaje), así como de la planificación, la elección de técnicas adecuadas y la evaluación de los resultados de aprendizaje (Gregorio Cano, 2014, p. 37).

En los últimos años, la investigación en didáctica de la traducción se ha ido afianzando, con el paso del tiempo, sobre todo en España, pero todavía se hace necesario incidir en la necesidad de estudios empíricos que arrojen cierta luz sobre cuestiones clave para el desarrollo de la teoría y la práctica que posibiliten avances en la formación de futuros traductores, así como en cuanto al reconocimiento de la disciplina a nivel científico y social (Marco como se citó en Gambier \& Van Doorslaer, 2009, p. 77).

Creemos que si uno de los objetivos de los planes de estudio de TI y de la didáctica de la traducción es el de formar futuros traductores, resulta lógico pensar, como apunta Mayoral (2001, p. 118), que si el aprendiz de traductor necesita rutinas de trabajo y toma de decisiones, así como distintos tipos de conocimiento, sobre los parámetros que afectan a la toma de decisiones o sobre los diferentes procedimientos o estrategias de traducción, entre otros; todo esto se aborde de manera más sistemática para que todos estos procesos se interioricen y preparar así a los futuros traductores en la labor que van a desarrollar una vez que hayan finalizado su período de formación académica. En este sentido, coincidimos con Hurtado Albir (2011) en la necesidad de desarrollar investigaciones empíricas enfocadas hacia el conocimiento de la adquisición de la CT y no limitarnos a meras especulaciones teóricas. Hurtado Albir apunta este déficit existente en cuanto a investigaciones empíricas se refiere como uno de los retos que tiene la Traductología y que en palabras de Hurtado Albir “[es] crucial para la didáctica de la traducción [...] investigar sobre la competencia traductora y su proceso de adquisición" (Hurtado Albir, 2011, p. 169).

A lo largo del desarrollo de nuestra investigación consideramos la CT como "el conjunto de capacidades, destrezas, conocimientos y actitudes necesarios para traducir, para lo que resulta fundamental el desarrollo de la capacidad de reconocimiento de problemas de traducción y de las estrategias para resolver dichos problemas" (Gregorio Cano, 2014, p. 43) y consideramos igualmente necesario que el desarrollo de la misma debe formar parte fundamental de los programas de formación de traductores. Además, compartimos las propuestas de los autores que apuntan que la CT se desglosa en una serie de componentes que están interrelacionados entre sí, donde la competencia estratégica ejerce de espina dorsal de la CT (Hurtado Albir, 2017; Kelly, 2002, 2005; PACTE, 2011a, 2011 b).

El estudio longitudinal que aquí presentamos se enmarca en el seno de una investigación más amplia, con una participación de más de 1.000 estudiantes de cinco centros de formación de traductores e intérpretes, que tenía como objetivo conocer y analizar la repercusión de los estudios universitarios en Traducción en España en el desarrollo de la competencia estratégica y, en particular, la capacidad de los estudiantes para identificar problemas de traducción mediante un estudio empírico, así como ahondar en el conocimiento del uso del metalenguaje que hacen los estudiantes de Traducción.

Una de las principales dificultades de la investigación que aquí se presenta es la falta de homogeneidad para denominar los diferentes conceptos de nuestra disciplina como, por ejemplo, a la 
hora de hablar de competencia traductora. En el caso de este concepto, en inglés, existen hasta siete denominaciones (Gregorio Cano, 2014). Esta falta de unanimidad en la conceptualización -no solo de denominación- existe en torno a aspectos básicos de la disciplina y, en particular, en el contexto de la formación de traductores, como en el caso de: ¿qué es un problema de traducción? ¿A qué nos referimos con "estrategia de traducción"? ¿Cómo describen los estudiantes los problemas de traducción que encuentran a la hora de realizar un encargo de traducción?

\section{Metalenguaje y “meta-debate” en Traductología.}

En la disciplina objeto de estudio no existe un consenso para la elaboración del metalenguaje inicial, entendido como un sistema de lenguaje que sirve de apoyo para construir otro sistema de conocimiento y lenguaje científico y que se utiliza para hablar y discutir sobre una disciplina, de cómo construirla, de los hechos básicos de los que se va a partir, de cómo definirlos, de las reglas deductivas que se fueran a utilizar (Mayoral, 2001, p. 66).

Coincidimos con Tang (2007, p. 359) en que el metalenguaje de la Traducción es mucho más que un aspecto terminológico, se trata de una conceptualización de una corriente cultural, académica y social de cada disciplina.

Los estudios de Traducción han heredado docenas de lenguajes de las diferentes disciplinas y escuelas en las que se funda y los intentos de normalizar conceptos y términos han sido escasos y terminan por añadir nuevas variantes al sistema al no contar con una aceptación lo suficientemente amplia (Mayoral, 2001, p. 67; Marco como se citó en Gambier \& Van Doorslaer, 2009, p. 76; SnellHornby como se citó en Gambier y Van Doorslaer, 2009, p. 123).

De esto se deduce, por tanto, que no existen definiciones universalmente aceptadas dentro de nuestro campo (Mayoral, 2001; Mayoral \& Díaz-Fouces, 2011) que se puedan tomar como punto de partida para entendernos entre nosotros, para que nos entiendan otros especialistas y para que sobre ellas se pueda vertebrar un aparato académico unívoco. Esta falta de consenso de la que hablamos puede ser fruto del grado de indefinición de nuestra disciplina (Mayoral, 2001, p. 47, p. 129, p. 134), por lo que cualquier avance en este sentido resultará clave de cara a la normalización terminológica y la formación de traductores al facilitar el proceso comunicativo (Mayoral, 2001, p. 118; Gambier como se citó en Gambier \& Van Doorslaer, 2009).

La relevancia del estudio que aquí se presenta, vital para la evolución en didáctica de la traducción (Gambier como se citó en Gambier \& Van Doorslaer, 2009, p.184), se encuentra en consonancia con Neubert y Shreve (1992, p. 11-12) quienes apuntaron que cuando todos hablemos la misma lengua, puede que lo que ahora parece ser un número infinito de enfoques de traducción termine por resultar realmente en unos pocos temas diferentes.

Sin embargo, a pesar de que el papel del metalenguaje en la disciplina de los Estudios de Traducción es de vital importancia, la atención que ha recibido por parte de los estudiosos de la misma desde una vertiente empírica ha sido muy limitada y desde 1972, cuando James Holmes propuso que 
se ahondara sobre la importancia del "meta-debate", pocos han sido los teóricos que han realizado aportaciones científicas al respecto (Delisle, Lee-Jahnke \& Cormier, 1999; Gambier \& Van Doorslaer, 2009; Tang, 2007). En este mismo sentido, el Dr. Frederic Chaume y la Dra. Anabel Borja, ponentes en el III Seminario de especialización en Didáctica de la traducción, celebrado en julio de 2016 en la Universidad Autónoma de Barcelona, apuntaron la importancia de que el estudiante de Traducción e Interpretación (TI) sea capaz y sepa hablar de sí mismo y de su disciplina de estudio y que, para ello, utilice el metalenguaje. Coincidimos con estos autores en que esta capacidad de hablar de uno mismo y de su campo de especialidad empodera al estudiante. Sin embargo, poco se ha avanzado en este sentido a partir de estudios empíricos que ahonden en esta cuestión.

La noción de problema de traducción. A la hora de realizar una traducción, el traductor experto o en formación, se encuentra con problemas que abordan de diferente manera a la hora de realizar un encargo de traducción (Gile, 2009; Shreve, 1997). No cabe ninguna duda de que en el entramado del proceso de la traducción, los problemas son uno de los elementos (casi) siempre presentes. Sin embargo, pese a la importancia de la que goza en Traducción la noción de problema y por extensión en didáctica, no existe una trayectoria de análisis exhaustiva en Traductología ni tampoco una definición de problema de traducción que goce de consenso general, al igual que tampoco contamos con una clasificación marco de los distintos tipos de problema de traducción que existen (Mayoral, 2001), hecho directamente relacionado con el metalenguaje y el objeto de nuestra investigación.

Si bien en Traductología carecemos de una trayectoria dilatada en el tiempo que investigue acerca de la noción de problema de traducción como eje central, aunque autoras como Nord (1991, 1997), Presas (1996), Orozco (2000) o Hurtado Albir (2011) sí han dedicado parte de su investigación a definir estos conceptos, la realidad es que estas investigaciones han sido casi anecdóticas y no han alcanzado una profunda repercusión en nuestra área de conocimiento. Esta falta de acuerdo se puede deber en parte a la alta complejidad que revierte en sí misma la noción problema de traducción y que, por tanto, necesita ser estudiada desde la vertiente empírica, para poseer un mejor conocimiento del concepto y por ende de la competencia traductora, hecho que tendría un efecto directo en la mejora de la didáctica de la traducción.

Por lo que respecta a la definición que adoptamos a lo largo de nuestra investigación, se trata de la definición propuesta por Hurtado Albir (2011, p. 286) a partir de la definición inicial de Nord (1991), de este modo, entendemos los problemas de traducción como “las dificultades (lingüísticas, extralingüísticas, etc.) de carácter objetivo con que puede encontrarse el traductor a la hora de realizar una tarea traductora". Si bien Hurtado Albir matiza que:

A la hora de definir los problemas de traducción y las dificultades ha de tenerse en cuenta en todo momento si el traductor es experto o en formación (y en qué nivel), ya que este hecho influirá inevitablemente en el proceso de sistematización de problemas de traducción y en los criterios de resolución de problemas (Hurtado Albir, 2011, p. 287).

Coincidimos con Hurtado Albir (2011) en que el interés por saber más sobre el problema de traducción goza de gran importancia desde el punto de vista de la didáctica de la traducción y de la evaluación de traducciones, ya que en palabras de Hurtado Albir (2011, p. 279): “[el problema de 
traducción] guía la elaboración de objetivos de aprendizaje, así como la comparación de la traducción con el texto original". Sin embargo, pese a ser una noción presente en la actividad cotidana de cualquier traductor experto o en formación, poco se ha avanzado en este sentido'.

La sensación que uno tiene al profundizar en las aportaciones de los diferentes autores que sí han focalizado su investigación en el tema que nos ocupa es que no se ha partido de lo que ya se había hecho, para otorgar de mayor significado a lo ya descubierto y, por lo tanto, complementar las investigaciones que se han llevado a cabo en este sentido y dotarlas de mayor significado y consistencia. En nuestro deseo por aportar a esta cuestión de gran importancia para la formación de futuros traductores, partiremos de las aportaciones con las que contamos en esta dirección, en un intento por ofrecer un marco para la sistematización del desarrollo de la capacidad de reconocer, así como en la manera de describir los diferentes problemas de traducción con los que se encuentran los estudiantes de traducción en diferentes momentos de su formación.

De este modo, a partir de las propuestas de Nord (1991) y PACTE (2011a, 2011b) y de los diferentes encuentros con el grupo de jueces expertos ${ }^{2}$ que colaboraron en nuestro estudio empírico, enunciamos la siguiente clasificación para los problemas de traducción (Gregorio Cano, 2014, pp. 130-131):

1. Problemas lingüísticos. Son problemas relacionados con el código lingüístico, fundamentalmente en el plano léxico (léxico no especializado) y en el morfosintáctico.

2. Problemas textuales. Son problemas relacionados con cuestiones de coherencia, progresión temática, cohesión, imágenes, tipologías textuales (convenciones de género), perspectiva, estilo, tono.

3. Problemas culturales. Son problemas que remiten a cuestiones culturales (presuposiciones), relacionadas con valores, mitos, percepciones, creencias y comportamientos y sus representaciones textuales, así como con las diferencias culturales de estos.

4. Problemas pragmáticos. Son problemas relacionados con la función del texto (escopo), la intención con la que se produce el texto, con los derivados del encargo de traducción, las características del receptor del texto, del contexto en el que se efectúa la traducción o del espacio y/o formato del texto.

Esta clasificación es la utilizada para el análisis de datos de la investigación empírica.

\section{OBJETIVO DE INVESTIGACIÓN}

La hipótesis general de la que partimos es que: la formación recibida en el seno de los estudios de Traducción e Interpretación favorece el desarrollo de la competencia traductora y, en particular, el de la competencia estratégica.

\footnotetext{
1 Las primeras aportaciones datan de 1988 (Nord) y, 25 años después, constatamos que no existe el consenso deseado en torno a esta cuestión, central para nuestra disciplina, síntoma tal vez de lo intermitente que han sido las investigaciones que se han llevado a cabo en este sentido.

${ }^{2}$ El grupo de jueces expertos juega un papel fundamental como red de apoyo en el diseño de este estudio empírico dada la cantidad de nociones y conceptos estudiados en el mismo que carecen de consenso en nuestra disciplina.
} 
Esta hipótesis se concreta en cuatro hipótesis específicas gracias a la existencia de un mismo grupo de estudiantes que conforman el estudio longitudinal. Estas hipótesis surgen como concreción del tema de investigación (Sierra, 1998, p. 70) y son fruto de la evolución de la propia investigación y nuestro deseo por dar respuesta a la cuestión investigada y confirmar (o no) una suposición inicial. Estas hipótesis específicas son:

1. Los estudiantes de TI próximos al momento de egreso son capaces de reconocer un mayor número de problemas de traducción que los estudiantes de TI en el momento de ingreso a los estudios.

2. La capacidad para reconocer y diferenciar entre problemas de traducción de diferente naturaleza de los estudiantes de TI próximos al momento de egreso es mayor que la de los estudiantes de Tl en el momento de ingreso a los estudios.

3. La capacidad de abstracción a la hora de identificar problemas de traducción de los estudiantes de Tl es mayor en el momento próximo al egreso que en el momento de ingreso a los estudios.

4. La riqueza del metalenguaje utilizado por los estudiantes de TI experimenta una evolución desde el momento de ingreso a los estudios de TI hasta el momento próximo al egreso.

Los resultados que se incluyen en este artículo responden a nuestro intento de confirmar o refutar las hipótesis a partir de las respuestas de los estudiantes que conforman el estudio longitudinal, al tratarse de los mismos estudiantes tanto en $1^{\circ}$ como en $4^{\circ}$, la hipótesis número 4.

Parece lógico pensar que si no existe un consenso en cuanto a las cuestiones teóricas que se pretenden analizar en nuestro estudio empírico, se pueda llegar a sembrar la duda de que los resultados gocen de relevancia; sin embargo, el hecho de que no exista un consenso, no quiere decir que en las aulas no se aborde nuestro objeto de estudio (el metalenguaje) de manera prácticamente diaria. Es obvio que en las clases de los centros de formación de traductores se enseña a reconocer y resolver un problema de traducción, para lo que primero se debe definir qué es y, por lo tanto, describirlo. Por todo lo anterior, con nuestro estudio no pretendemos la matematización de una realidad tan compleja como es el desarrollo de la competencia traductora como producto de la formación formal recibida en el seno de un centro de educación superior, ya que ni el currículum, ni el perfil de los estudiantes, ni la TI son realidades objetivas. Sin embargo, lo que sí pretende nuestra investigación es servir como ejercicio de cientificidad como refuerzo para identificar la realidad objeto de nuestro estudio y reafirmar la necesidad de un compendio de términos y conceptos que faciliten el desarrollo de la competencia traductora como parte de las rutinas de aprendizaje. Partimos de la premisa de que el metalenguaje variará dependiendo del enfoque teórico, la escuela, así como la combinación lingüística (Delisle et al., 1999; Gambier como se citó en Gambier \& Van Doorslaer, 2009; Mayoral, 1999; Snell-Hornby como se citó en Gambier \& Van Doorslaer, 2009; Van Vaerenbergh como se citó en Gambier \& Van Doorslaer, 2009).

\section{MÉTODO}

Los datos que se analizan en el presente artículo se extraen de una de las herramientas (Gregorio Cano, 2014) que se diseñaron en el proyecto de estudio del desarrollo de la competencia estratégica, para lo que se combinaron enfoques cuantitativos y cualitativos (enfoque mixto). 
A continuación describimos brevemente las bases metodológicas de la investigación llevada a cabo: el diseño, las fases, las estrategias de obtención de datos utilizadas, la población objeto de estudio y el proceso que ha girado en torno al estudio empírico, así como las pautas seguidas para el análisis de los datos que se presentan en el presente artículo.

\section{Diseño}

La herramienta en cuestión, diseñada en papel, consta de dos partes: la primera se centra en los datos sociodemográficos ("perfil del estudiante"), donde se registran datos como lenguas de trabajo, experiencias interculturales o el método de adquisición de los conocimientos de lengua extranjera, entre otros aspectos (Gregorio, en prensa). La segunda parte consta de un encargo de pre-traducción y una serie de preguntas abiertas relacionadas con el encargo. Los resultados que se presentan en este artículo se corresponden con la segunda parte de la herramienta.

Cabe señalar que entre las razones principales que motivaron la elección del diseño de la herramienta en papel están las ventajas que ofrece, desde el punto de vista de la obtención de datos, la sistematización y el gran número de sujetos a los que se puede acceder en cada aplicación (poblaciones cautivas, García Ferrando 2005, p. 180), así como la posibilidad de que el investigador exponga brevemente los objetivos y el protocolo de cumplimentación de la herramienta al principio de cada sesión de recogida de datos y poder resolver cualquier incidencia durante dicha fase de recogida de datos ${ }^{3}$.

\section{Participantes}

Decidimos aplicar la estrategia más utilizada en aquellos casos en los que no es abordable la totalidad de la población; para ello se escogió una porción de la población, conocida como muestra (Sierra, 1998, p. 179), que comparte las características poblacionales objeto de investigación (Gregorio Cano, 2014, p. 104).

No se puede perder de vista que nuestro estudio no es experimental, sino exploratorio, hecho que influye de manera determinante en el tratamiento que se realiza de la muestra, así como para el análisis de los datos. Una vez establecida que la representatividad de la muestra no es estadística, se hace indispensable comprender que el muestreo definido se decidió que se haría desde una perspectiva no probabilística (Fink, 2003, p. 16; Rojas, Fernández \& Pérez, 1998, p. 65).

Con el objetivo de garantizar una mayor representatividad en la muestra y dado que contar con la participación de todos los centros de TI de España no era una posibilidad, planificamos la elección de los centros de acuerdo con una serie de criterios (Gregorio Cano, 2014, pp. 104-105) que se pueden resumir en:

1. Nuestro deseo de estudiar varios centros españoles con diferentes características, ubicados en diferentes áreas geográficas de España para no focalizar nuestro estudio en un área geográfica exclusiva (norte o sur, este u oeste).

\footnotetext{
${ }^{3}$ A la hora de solicitar la participación de los estudiantes, la investigadora explicaba el uso que se haría de los datos (anónimos), así como solicitaba la firma del consentimiento informado que rezaba "he sido puntualmente informado de los objetivos de este proyecto de investigación y acepto participar y colaborar en él".
} 
2. La inclusión de centros españoles que cuenten con un volumen de estudiantes diferente (reducido, intermedio y elevado).

3. El estatus de público o privado de los centros.

4. La oferta de lenguas $A$ y $B$.

5. La viabilidad de acceso a la hora de planificar la fase de recogida de datos de nuestro estudio empírico.

De este modo, los centros de formación de traductores participantes en nuestro estudio fueron la Universidad de Granada (UGR), la Universidad de Salamanca (USAL), la Universitat Jaume I (UJI), la Universidad Pablo de Olavide (UPO) y la Universidad Pontificia de Comillas (UPCO). En cuanto a los criterios de inclusión de los estudiantes participantes responden en todo momento a los objetivos perseguidos, por lo que la muestra de nuestro estudio cuenta con estudiantes próximos al momento de ingreso $\left(1^{\circ}\right)$ a los estudios de $\mathrm{TI}$ y al momento de egreso ( $\left.4^{\circ}\right)$.

Perfil de la muestra. La muestra del estudio de casos múltiple la componen 37 estudiantes de la UGR, que resultó como un subproducto espontáneo de nuestra investigación una vez que cruzamos los documentos de identidad de la muestra total de nuestra investigación (1.046 estudiantes) de $1^{\circ} \mathrm{y}$ $4^{\circ}$. El hecho de que la población de nuestro estudio longitudinal proceda en su totalidad de la UGR se puede entender si tenemos en cuenta que es la universidad que cuenta con una mayor representación en nuestro macro-estudio tanto en $1^{\circ}$ (38,6\%, 253 estudiantes) como en $4^{\circ}$ (40,2\%, 157 estudiantes).

Edad de los estudiantes participantes. Como era de esperar tras los resultados obtenidos en el macro-estudio (Gregorio, en prensa), el rango de edad más frecuente de los estudiantes del longitudinal en $1^{\circ}$ es el que comprende el rango de edad de los 17 a los 20 años, edad que tienen 36 de ellos, mientras que solo uno tiene más de 21 años. Como cabe esperar al tratarse de los mismos estudiantes cuatro años más tarde, en $4^{\circ}$, la edad de los estudiantes se sitúa en el rango de edad de entre 21 y 24 años para 36 de ellos, y en el de entre 25 y 28 años para uno de ellos.

Sexo de los estudiantes participantes. En cuanto al sexo de la muestra del estudio longitudinal contamos con 27 mujeres y con 10 hombres, que a su vez coincide con la distribución mayoritaria de mujeres frente a hombres del macro-estudio:

Lenguas $B$ y $C^{4}$. De los 37 estudiantes que conforman la muestra contamos con tres de las cuatro lenguas B que oferta la FTI de la UGR: 25 con lengua B inglés, 9 con lengua B francés y 3 con lengua $B$ alemán. Los porcentajes de participación de los estudiantes se corresponden con la proporción de estudiantes por lengua B del macro-estudio (Gregorio, en prensa), donde tanto en $1^{\circ}$ como en $4^{\circ}$ contamos con una mayor participación de estudiantes con lengua $B$ inglés, seguidos de los estudiantes con lengua $B$ francés y, por último, los estudiantes con lengua B alemán ${ }^{5}$ :

\footnotetext{
${ }^{4}$ El uso de las denominaciones de lengua $A$, lengua $B$ y lengua $C$ se corresponde, en términos generales, con las lenguas de trabajo de los futuros traductores, a saber: lengua $A$ en este estudio es el español, la lengua B es la primera lengua extranjera y la lengua $C$ es la segunda lengua extranjera.

${ }^{5}$ Nuestro estudio longitudinal no cuenta con ningún estudiante con lengua B árabe, ya que no obtuvimos ninguna correspondencia entre estudiantes de $1^{\circ}$ y $4^{\circ}$ que hubiesen cumplimentado nuestra herramienta en los dos momentos de recogida de datos de nuestro estudio.
} 
Tabla 1

Combinación lingüística de los estudiantes participantes

\section{Combinación lingüística}

\begin{tabular}{|c|c|c|c|c|c|c|c|}
\hline \multicolumn{4}{|c|}{ Primer año } & \multicolumn{4}{|c|}{ Cuarto año } \\
\hline \multirow{2}{*}{$\begin{array}{c}\text { Lengua } \\
\text { C }\end{array}$} & \multicolumn{3}{|c|}{ Lengua $B$} & Lengua & \multicolumn{3}{|c|}{ Lengua $B$} \\
\hline & alemán & francés & inglés & $c$ & alemán & francés & inglés \\
\hline alemán & & $\begin{array}{c}3 \\
(33,33 \%)\end{array}$ & $\begin{array}{c}5 \\
(20 \%)\end{array}$ & alemán & & $\begin{array}{c}3 \\
(33,33 \%)\end{array}$ & $\begin{array}{c}7 \\
(28 \%)\end{array}$ \\
\hline chino & & & $\begin{array}{c}4 \\
(16 \%)\end{array}$ & chino & & & $\begin{array}{c}5 \\
(20 \%)\end{array}$ \\
\hline francés & & & $\begin{array}{c}15 \\
(60 \%)\end{array}$ & francés & & & $\begin{array}{c}12 \\
(48 \%)\end{array}$ \\
\hline inglés & $\begin{array}{c}2 \\
(66,66 \% \%)\end{array}$ & $\begin{array}{c}6 \\
(66,67 \%)\end{array}$ & & inglés & $\begin{array}{c}2 \\
(66,66 \%)\end{array}$ & $\begin{array}{c}6 \\
(66,67 \%)\end{array}$ & \\
\hline ruso & $\begin{array}{c}1 \\
(33,34 \%)\end{array}$ & & $\begin{array}{c}1 \\
(4 \%)\end{array}$ & ruso & $\begin{array}{c}1 \\
(33,34 \%)\end{array}$ & & $\begin{array}{c}1 \\
(4 \%)\end{array}$ \\
\hline total & $3(8,12 \%)$ & $9(24,32 \%)$ & $25(67,56 \%)$ & total & $3(8,12 \%)$ & $9(24,32 \%)$ & $25(67,56 \%)$ \\
\hline
\end{tabular}

Si prestamos atención al número de estudiantes en cada una de las lenguas $C$ presentes en nuestro estudio, se observa que algunos estudiantes cambiaron su combinación lingüística desde $1^{\circ}$ a $4^{\circ}$, como es el caso de 3 de ellos que cambiaron su lengua $C$ francés por alemán (dos de ellos) y por chino (un estudiante).

\section{Técnicas de recolección de datos}

La elección del texto que se incluye en la tarea responde a un proceso de reflexión y búsqueda de la idoneidad del género textual (García Izquierdo, 2000, 2012; Elena, 2011; Roiss, 2006a, 2006b), clave para el objeto de investigación de nuestro trabajo. La razón por la que el texto incluido en la herramienta está escrito en español se debe a que es lengua oficial de los centros en los que se desarrolló la investigación y a nuestro objetivo específico de estudiar la capacidad de los estudiantes para reconocer y describir (no se pedía en ningún momento la traducción) los problemas de traducción planteados por el texto. Asimismo, la elección de la lengua origen del texto encuentra su justificación en la dificultad añadida que habría entrañado encontrar un texto similar con el mismo tipo de problemas en todas las lenguas $B$ y que las respuestas de los estudiantes respondieran a un texto con características y problemas similares, de ahí que finalmente se optara por la selección de un texto en lengua A español, lengua oficial para todos los participantes en nuestro estudio. Somos conscientes de que el hecho de que se trate de un encargo de traducción de la lengua A hacia la B podría suponer mayor dificultad a la hora de expresar los diferentes aspectos del texto, pero consideramos que facilita la comprensión del texto, además de minimizar las posibles dificultades derivadas del nivel de lengua $B$ de los participantes en el estudio (Kelly, 2005, p. 139-140). 
En resumen, la tarea consiste en un ejercicio de pre-traducción, el mismo tanto para los estudiantes de $1^{\circ}$ como para los de $4^{\circ}$, que incluye un encargo de traducción que reza: “Este texto pertenece a la revista de vuelo de una línea aérea. Imagina que se te ha encargado que lo traduzcas a tu lengua $B$ para su publicación en la misma revista. Léelo con atención y pasa a la página siguiente".

Inmediatamente después del texto, aparecen las instrucciones concretas con lo que los estudiantes debían hacer una vez que hubiesen leído el texto, así como el espacio reservado para que respondieran. En la primera parte del encargo se ofrece una breve contextualización sobre dónde se va a publicar la traducción y la combinación lingüística. En la segunda parte se enfatiza en qué deben hacer los estudiantes en concreto: "no hay que traducir el texto, solo escribir los problemas que cada estudiante identifica (hasta cinco), así como describir cómo los resolverías".

\section{Tabla 2}

Texto del encargo de pre-traducción

\section{TAL COMO SOMOS}

El vermú de los domingos

tomando el vermú en Zahara de la Sierra (Cádiz)

¿Qué hacen los españoles el domingo por la mañana? Tomar el aperitivo, también llamado el vermú. Para los españoles la mañana es aquel espacio de tiempo que finaliza a la hora de comer, que en este caso y además en domingo, se enlaza con el café y las pastas de la merienda sin darse uno cuenta. El vermú o el aperitivo es un acto social abierto a todas las edades y condiciones: padres llevan a sus retoños, los mayores se encuentran con sus hijos, vecinos con vecinos, jefes con empleados y amigos con enemigos. Todos en el mismo bar, más o menos entre la 1 y las 3. El bar es el de toda la vida, sin moderneces, con el mismo Paco, Antoni o Patxi en la barra que además, por suerte, está normalmente a tiro de piedra del portal de tu casa. Con tanto vecino, cuñado y amigo, tres o cuatro rondas no te las quita nadie. Menos mal que la ingesta alcohólica viene acompañada de ese fabuloso invento que es la tapa. Boquerones en vinagre, queso, croquetas, gambas con gabardina y un largo etcétera de variedades regionales. Una delicia de domingo.

Como se desprende de la lectura del texto, hay numerosos problemas de traducción, de ahí que los estudiantes pudieran señalar hasta un máximo de $\operatorname{cinco}^{6}$, sin necesidad de que incluyeran la traducción, ya que el interés de nuestro estudio no se centra en la evaluación de las traducciones (producto) ${ }^{7}$, en torno al que se han articulado muchas investigaciones a lo largo del tiempo (García de Toro \& García Izquierdo, p. 16), sino en conocer el proceso y la capacidad de los estudiantes en $1^{\circ}$ y $4^{\circ}$ a la hora de identificar los distintos tipos de problemas existentes, así como analizar cómo lo describen.

\footnotetext{
${ }^{6}$ En este sentido, nuestro trabajo coincide parcialmente con la propuesta de ejercicio de la ficha de trabajo de La Rocca (2007, p. 221).

7 Somos conscientes de que el hecho de no haber solicitado la traducción del texto conlleva una serie de limitaciones intrínsecas para nuestra investigación, ya que en numerosas ocasiones el análisis que se realiza previamente a la traducción deja al margen algunos problemas que se pueden percibir solo a la hora de realizar la traducción en cuestión.
} 
El texto incluido en nuestra herramienta contaba con una imagen (elemento no verbal) y fue sometido al análisis pormenorizado por un grupo de jueces expertos ${ }^{8}$ con el objetivo de detectar y establecer los problemas de traducción existentes en el mismo y que serían objeto de análisis. La categorización de los distintos problemas de traducción que existen en el texto fue fruto de varios encuentros en grupo e individuales de la investigadora con cada experto. Por todo ello, a consecuencia de las reuniones con el grupo de expertos, y del pre-análisis de los datos con los que contamos, los resultados los presentaremos en torno a cuatro ejes principales que engloban los problemas de traducción detectados en el texto incluido en la herramienta por el grupo de expertos, a saber:

\section{Tabla 3}

Combinación lingüística de los estudiantes participantes

Tipos

Problemas lingüísticos

Problemas culturales

Problemas pragmáticos

\section{Problemas}

1. Léxico.

2. Expresiones idiomáticas

3. Sintaxis.

1. Perspectiva del texto.

2. Estilo.

3. Tono del texto.

4. Imagen.

1. Comida

2. Concepto "bar".

3. Nombres propios:

4. Relaciones sociales:

5. Tiempo.

6. Espacio.

1. Función.

2. Intención

3. Encargo de traducción.

4. Lector.

5. Formato y/o espacio del texto.

Criterios de calidad de la herramienta. Nuestra herramienta cuenta con criterios de calidad que responden a dos aspectos fundamentales: la fiabilidad (Buendía \& Colás, 1998, p. 240; Buendía, Colás \& Hernández, 1998, p. 146) y la validez de la misma (Buendía \& Colás, 1998, p. 329; Sierra, 1998, p. 322)."

A la hora de diseñar la herramienta seguimos una serie de pasos con el fin de que la herramienta fuese fiable, a saber:

- Control de las preguntas incluidas en la herramienta, para garantizar que el número de ítems era apropiado.

\footnotetext{
${ }^{8}$ El grupo de expertos lo conformaron la Dra. Barabino, la Dra. Calvo, la Dra. Gregorio, la Dra. Kelly, el Dr. Mayoral y la Dra. Way.
} 
- Pilotaje en una muestra de estudiantes de similares características a los estudiantes que conforman la muestra final de la investigación.

- Revisión de la herramienta en distintos momentos por un grupo de jueces expertos.

- Recogida de datos en el mismo período del curso a todos los estudiantes y con la misma información para poder cumplimentarlo. Se diseñó un protocolo que fue leído a la hora de administrar el cuestionario y, de ese modo, garantizar que la información disponible por los estudiantes fuesen siempre la misma (Gregorio Cano, en prensa).

En cuanto a la validez, seguimos las recomendaciones de Buendía y Colás y Sierra para obtener validez de contenido, de constructo y de criterio (Gregorio, en prensa).

\section{Procedimiento}

La herramienta se administró en dos momentos de la formación de los estudiantes: en las primeras semanas de primer año (momento cercano al ingreso) y en las últimas semanas del cuarto de los estudios (momento próximo al egreso).

Pre-análisis de los datos: el estudio longitudinal. El estudio longitudinal se corresponde con un grupo relativamente grande de estudiantes para un estudio de casos, en total 37, que cumplimentaron nuestra herramienta en los dos momentos de la formación objeto de nuestro estudio: en $1^{\circ}$ y en $4^{\circ}$, cuatro años más tarde. El principal objetivo que perseguimos con este estudio longitudinal es intentar establecer tendencias más concretas que las obtenidas en el macro-estudio (correspondientes a los datos de los 1.046 estudiantes), al trabajar con los mismos estudiantes en la primera toma de contacto con los estudios de $\mathrm{TI}$ y con sus últimas experiencias como futuros traductores y/o intérpretes a punto de finalizar su formación.

\section{Análisis de los datos}

Como ya hemos apuntado, los datos de nuestra investigación se encontraban en formato papel, por lo que se hizo necesario sistematizar previamente al análisis manualmente los datos -en papel- de nuestra herramienta a un formato procesable por el paquete de datos SPSS, es decir, disponer los datos en forma numérica y digital. Al igual que el resto de datos, los correspondientes a la pregunta con respuesta abierta se encontraban manuscritos por los estudiantes, por lo que se procedió a la digitalización de todas las respuestas de los participantes. El proceso de digitalización fue llevado a cabo por un solo investigador.

Los datos cualitativos y el análisis de estos han representado un proceso de reflexión constante con el fin de encontrar la vía más apropiada para garantizar unos resultados objetivos y representativos de la realidad estudiada. El análisis de la evolución de los estudiantes que componen el estudio longitudinal se llevó a cabo por medio de un estudio de casos, ya que este método permite el análisis de los casos en profundidad, en tanto que se trata de un enfoque eminentemente cualitativo y busca comprender el proceso por el que tienen lugar ciertos fenómenos (Rialp, Martínez \& Rialp, 2005, p. 100). 
Los resultados de nuestra investigación de naturaleza descriptiva y cualitativa arrojan cierta luz y significado a una cuestión fundamental, al mismo tiempo que densa y poco estudiada desde una perspectiva aplicada-empírica: el uso del metalenguaje por los traductores en formación. Por tanto, somos conscientes de que, dada la no univocidad de los conceptos aquí presentados, estos no están exentos de debate y queremos puntualizar que el significado de nuestros resultados se construye a partir de las percepciones de sus actores. Los resultados obtenidos gracias a nuestra investigación recogen tendencias y percepciones aplicables a nuestra muestra final de participantes y en ningún caso son extrapolables al universo hipotético o población total (Sierra, 1998, p. 179).

\section{RESULTADOS}

En este artículo presentamos solo una selección de los datos que conforman el estudio longitudinal, dada la extensión que alcanzó el estudio intensivo de casos, a saber: el perfil de la muestra y una selección del uso del metalenguaje utilizado a la hora de describir los distintos problemas de traducción presentes en el encargo de pre-traducción incluido en nuestra herramienta. En algunos apartados hacemos referencia a ciertos resultados del macro-estudio para contextualizar algunos de los datos obtenidos en el estudio longitudinal por ser complementarios.

\section{Detección de problemas y descripción: el uso del metalenguaje}

Los criterios que se han tenido en cuenta de cara a determinar si los distintos participantes de nuestra investigación experimentan una evolución en cuanto al uso del metalenguaje para describir los problemas de traducción presentes en el texto se resumen en la siguiente tabla:

\section{Tabla 4}

Criterios seguidos para realizar el recuento de los estudiantes que evolucionan en cuanto al uso del metalenguaje

Con el objetivo de describir las tendencias de los estudiantes, centramos nuestra atención en la manera de describir los distintos problemas presentes en el texto en los dos momentos de formación objeto de nuestro estudio $\left(1^{\circ}\right.$ y $\left.4^{\circ}\right)$ : los términos que utilizan para referirse a los distintos conceptos, comprobar si incluyen en sus descripciones conceptos propios de la TI como pueden ser: lengua origen, encargo, receptor, lector, referencia cultural, por mencionar solo algunos ejemplos.

El número de estudiantes que evolucionan de $1^{\circ}$ a $4^{\circ}$ respecto de este parámetro de estudio son 22 , es decir, el 59,5\% (22 estudiantes) de la muestra, frente al 40,5\% que de acuerdo con las respuestas de los estudiantes no parecen contar con un abanico de términos o conceptos del metalenguaje de nuestra disciplina?.

\footnotetext{
? Los resultados que se presentan aquí se han extraído del estudio de casos exhaustivo.
} 
A continuación los ejemplos del metalenguaje utilizado por los participantes de nuestro estudio longitudinal con el fin de ofrecer una visión global del uso del metalenguaje entre los estudiantes en el momento de ingreso y en el momento de egreso, tras cuatro años de formación especializada en TI. Asimismo, cabe destacar que en las páginas que aparecen a continuación se presentarán los argumentos aportados por los estudiantes ${ }^{10}$, categorizados y sin especificar qué estudiante ha dicho uno u otro argumento, en una versión que hemos denominado "deshidratada", con el objetivo de facilitar el análisis del metalenguaje por tratarse de unidades de significado más simples que los argumentos originales -completos-de los estudiantes ${ }^{11}$. Como se puede observar, ordenamos los diferentes ejemplos de acuerdo con la clasificación de problemas de traducción que hemos propuesto.

Aunque el análisis que aquí se presenta se corresponde con las respuestas de los 37 participantes del estudio longitudinal en $1^{\circ}$ y en $4^{\circ}$, y la naturaleza de los argumentos y del análisis sea de corte cualitativo, creemos que los apuntes cuantitativos que incluimos en algunos momentos pueden resultar claves para sustentar nuestra investigación cualitativa.

Problemas lingüísticos. Son aquellos relacionados con el código lingüístico, fundamentalmente en el plano léxico (léxico no especializado) y morfosintáctico. Como puede apreciarse en la tabla que mostramos a continuación, la inmensa mayoría de estudiantes recogen entre sus respuestas problemas lingüísticos en ambos momentos formativos objeto de estudio ${ }^{12}$ :

\section{Tabla 5}

Número de estudiantes que reconocen problemas lingüísticos

\begin{tabular}{|c|c|c|c|c|}
\hline & Frecuencia & $\%$ válido & Frecuencia & \% válido \\
\hline & \multicolumn{2}{|c|}{10} & \multicolumn{2}{|c|}{$4^{\circ}$} \\
\hline Sí & 37 & $100 \%$ & 35 & $94,59 \%$ \\
\hline No & & & 2 & $5,41 \%$ \\
\hline total & 37 & $100 \%$ & 37 & $100 \%$ \\
\hline
\end{tabular}

Según estos datos, resulta especialmente interesante que no existan prácticamente diferencias entre el porcentaje de estudiantes de un grupo poblacional y otro con respecto a este tipo de problemas.

Cabe hacer un inciso para ejemplificar el tipo de respuesta aportada por los estudiantes a la hora de explicitar los problemas de este tipo. De este modo, a la hora de reducir la información cualitativa, encontramos que los estudiantes describen los problemas lingüísticos en torno a los siguientes

\footnotetext{
10 Analizamos de manera global del metalenguaje utilizado en $1^{\circ}$ y en $4^{\circ}$, no estudiante por estudiante, con el objetivo de identificar tendencias y diferencias en cuanto al metalenguaje utilizado por los dos grupos poblacionales incluidos en nuestra investigación.

11 En caso de querer acceder a los argumentos completos, estos se encuentran en Gregorio Cano (2014).

12 En este sentido, la tendencia de los estudiantes hacia la palabra no es un hecho aislado de los participantes del estudio longitudinal, ya que los resultados del macro-estudio constatan esta tendencia con el $61,8 \%$ (405 estudiantes) en $1{ }^{\circ}$ y el $56,5 \%$ ( 221 estudiantes) en $4^{\circ}$ que también detectaron problemas lingüísticos relacionados con el vocabulario del texto.
} 
conceptos principalmente: término(s), palabra(s), vocabulario-léxico, frases hechas/expresiones, nombres (de comidas) /platos.

A continuación aparecen el número de estudiantes y el porcentaje de respuesta para las cinco categorías que el grupo de expertos apuntó como susceptibles de ser reconocidas por los estudiantes. Observamos que más de la mitad de los estudiantes en ambos momentos de su formación tiene problemas con el vocabulario que aparece en el texto, como, por ejemplo, con las palabras: retoños, merienda, tapa, ronda, por mencionar solo algunas. Por otra parte, los estudiantes señalan en ambos momentos de su formación tener en distintas proporciones problemas con otros aspectos, como con los nombres de los platos, el $32,43 \%$ en $1^{\circ}$ y el $54,05 \%$ en $4^{\circ}$. Asimismo, otro de los problemas en el plano léxico apuntado en un porcentaje bastante alto se corresponde con las expresiones o frases hechas, señalado por el $75,67 \%$ en $1^{\circ}$ y por el $48,64 \%$ en $4^{\circ}$. Por último, pero en menor medida, los estudiantes de ambos grupos poblacionales incluyen entre los problemas lingüísticos los relacionados con cuestiones sintácticas y con cuestiones de ortotipografía en ambos momentos formativos:

\section{Tabla 6}

Número y tipo de problemas lingüísticos señalados por los estudiantes

\section{Primer año}

Lingüísticos

\begin{tabular}{|l|}
\hline \multicolumn{1}{|l|}{ Lingüísticos } \\
\hline
\end{tabular}

Total señalado Frecuencia \%
$89,18 \%$

$32,43 \%$

12

28

$75,67 \%$

$8,1 \%$

$5,4 \%$

37
25

$3 \quad 8,1 \% \quad 34$

35 no señalado
Cuarto año

señalado no señalado
Frecuencia \% Frecuencia \%

$20 \quad 54,05 \%$

17

$45,95 \%$

$67,57 \%$

$20 \quad 54,05 \%$

17

$45,95 \%$

$10,82 \%$

$24,33 \%$

18

$48,64 \%$

19

$51,36 \%$

$91,9 \%$

5

$13,51 \%$

32

$86,49 \%$

$94,6 \%$

$2,7 \%$

36

$97,3 \%$

$100 \%$

37

$100 \%$

De esta tabla se desprende que en primero $\left(1^{\circ}\right)$ los estudiantes muestran una tendencia a la palabra similar a la que tienen en cuarto $\left(4^{\circ}\right)$ y no se pueden apreciar diferencias sustanciales entre ambos grupos poblacionales, salvo por el apartado que alude a los problemas lingüísticos derivados de los nombres de los platos que identifican un mayor número de estudiantes de $4^{\circ}$. Este hecho tal vez pueda estar motivado por la experiencia que hayan tenido durante la carrera con la traducción de este tipo de textos, frecuentes en el aula de traducción. 
Por último, y con el fin de dotar de mayor sentido a los datos estadísticos, incluimos algunos ejemplos por categoría con el tipo de metalenguaje utilizado por los estudiantes de ambos grupos poblacionales que abordan cada una de las categorías:

\section{Tabla 7}

Ejemplos del metalenguaje utilizado para los problemas lingüísticos

\section{Ejemplos}

10 $4^{\circ}$

Término muy español.

Término bastante extendido.

No es un término que existe o tenga que existir en otras lenguas. Terminología específica. Problema que no es de gran complicación. El término no es conocido. Término merienda. Términos muy típicos.

Término que puede causar confusión.

Es una palabra muy coloquial española.

Problemas en cuanto al léxico.

La traducción del nombre de las tapas. Los nombres de los platos siempre plantean un problema de traducción

Problema de las expresiones idiomáticas en español.

Frases hechas.

Expresión hecha española.

\section{lingüístico:}

frases hechas

\begin{tabular}{|l|l|}
\hline & $\begin{array}{l}\text { Iiteralmente. } \\
\text { Idioms como “a tiro de piedra”. }\end{array}$ \\
\hline $\begin{array}{l}\text { Hay algunos tipos de estructuras semánticas o } \\
\text { sintácticas que pueden suponer un problema. } \\
\text { Esta frase no se puede traducir literalmente. } \\
\text { Estructuras gramaticales que desconozco. } \\
\text { Esta es una forma de hablar muy española y no } \\
\text { podríamos traducir esta frase. }\end{array}$ \\
\hline \multirow{2}{*}{ ortotipografía } & $\begin{array}{l}\text { La hora es escribiendo } 13 \text { cuando son las } 1 \text { p.m. } \\
\text { El título debe comenzar con mayúscula. }\end{array}$ \\
\hline
\end{tabular}

Frases hechas que no se pueden traducir de forma literal.

Expresión idiomática que no se puede traducir literalmente.

Cambiaría el orden de la frase.

“Vermú” es la castellanización del término "vermouth".

Problemas textuales. En esta categoría se recogen los argumentos relacionados con cuestiones de coherencia, progresión temática, cohesión, imágenes, tipologías textuales (convenciones de género), estilo o tono. El $5.4 \%$ de los estudiantes en $1^{\circ}$ señaló algún problema de esta naturaleza, mientras que en $4^{\circ}$ el porcentaje fue algo mayor con el $13.51 \%$ : 


\section{Tabla 8}

Número de estudiantes que reconocen problemas textuales

\begin{tabular}{|c|c|c|c|c|}
\hline & Frecuencia & $\%$ válido & Frecuencia & $\%$ válido \\
\hline & \multicolumn{2}{|c|}{10} & \multicolumn{2}{|c|}{$4^{0}$} \\
\hline Sí & 2 & $5.4 \%$ & 5 & $13.51 \%$ \\
\hline No & 35 & $94.59 \%$ & 32 & $86.49 \%$ \\
\hline total & 37 & $100 \%$ & 37 & $100 \%$ \\
\hline
\end{tabular}

Como puede apreciarse en la tabla 9, los problemas textuales no son los más reconocidos por los participantes en el estudio longitudinal, al igual que tampoco lo fueron en el macro-estudio (Gregorio Cano, 2014):

\section{Tabla 9}

Número y tipo de problemas textuales señalados por los estudiantes

\begin{tabular}{|c|c|c|c|c|c|c|c|c|}
\hline \multirow{3}{*}{$\begin{array}{c}\text { Problemas } \\
\text { textuales }\end{array}$} & \multicolumn{4}{|c|}{ Primer año } & \multicolumn{4}{|c|}{ Cuarto año } \\
\hline & \multicolumn{2}{|c|}{ señalado } & \multicolumn{2}{|c|}{ no señalado } & \multicolumn{2}{|c|}{ señalado } & \multicolumn{2}{|c|}{ no señalado } \\
\hline & Frecuencia & $\%$ & Frecuencia & $\%$ & Frecuencia & $\%$ & Frecuencia & $\%$ \\
\hline tono & $N / A^{13}$ & $\mathrm{~N} / \mathrm{A}$ & 37 & $100 \%$ & 1 & $2,7 \%$ & 36 & $97,3 \%$ \\
\hline perspectiva & 2 & $5,4 \%$ & 35 & $94,6 \%$ & N/A & N/A & 37 & $100 \%$ \\
\hline estilo & 2 & $5,4 \%$ & 35 & $94,6 \%$ & 2 & $5,4 \%$ & 35 & $94,6 \%$ \\
\hline Total & \multicolumn{2}{|l|}{37.} & \multicolumn{2}{|c|}{$100 \%$} & 37 & & \multicolumn{2}{|c|}{$100 \%$} \\
\hline
\end{tabular}

Así como hemos hecho en el caso de los problemas lingüísticos, a continuación incluimos los argumentos correspondientes a los problemas textuales para mostrar el tipo de respuesta aportada por los participantes en la investigación:

\section{Tabla 10}

Ejemplos de los argumentos que apuntan problemas textuales

\begin{tabular}{|c|c|c|}
\hline Ejemplos & 10 & $4^{0}$ \\
\hline tono & $\begin{array}{l}\text { Ningún estudiante en primero ha encontrado } \\
\text { problemas de este tipo. }\end{array}$ & Tener en cuenta el tono del texto. \\
\hline perspectiva & El artículo está planteado para españoles. & $\begin{array}{l}\text { Ningún estudiante en cuarto ha } \\
\text { encontrado problemas de este tipo. }\end{array}$ \\
\hline estilo & $\begin{array}{l}\text { Utiliza un estilo informal. } \\
\text { Alguna expresión que destaque el estilo (expresiones } \\
\text { como “a tiro de piedra”. Intenta buscar alguna } \\
\text { expresión que destaque del estilo). }\end{array}$ & Mantener el estilo informal. \\
\hline
\end{tabular}

13 Utilizamos N/A (no apuntado) en aquellos casos en los que no se ha señalado ningún problema de esta naturaleza. 
Problemas culturales. En esta categoría se enmarcan los problemas que remiten a cuestiones temáticas (conceptos especializados), enciclopédicas, culturales (presuposiciones). El número de estudiantes que reconoce problemas de este tipo en cada uno de los momentos objeto de estudio queda distribuido de la siguiente manera:

\section{Tabla 11}

Número de estudiantes que reconocen problemas culturales

\begin{tabular}{|c|c|c|c|c|}
\hline & Frecuencia & \% válido & Frecuencia & \% válido \\
\hline & \multicolumn{2}{|c|}{10} & \multicolumn{2}{|c|}{$4^{\circ}$} \\
\hline Sí & 29 & $78,37 \%$ & 26 & $70,27 \%$ \\
\hline No & 8 & $21,63 \%$ & 11 & $29,73 \%$ \\
\hline total & 37 & $100 \%$ & 37 & $100 \%$ \\
\hline
\end{tabular}

Al igual que en el caso de los problemas lingüísticos, a continuación incluimos algunas de las palabras y de los conceptos más repetidos a la hora de describir los problemas relacionados con los problemas de naturaleza cultural. De este modo, tanto los estudiantes en primero como en cuarto han utilizado expresiones tales como: problema cultural, referencia cultural, costumbre, típico, España, para referirse a los distintos problemas culturales que presentaba el texto.

Si recordamos las categorías apuntadas por el grupo de expertos en lo relativo a las presuposiciones relacionadas con la comida, estas eran: tapa, concepto comida, platos (variedad regional) y merienda. En cuanto a las presuposiciones culturales relacionadas con el concepto bar, el grupo de expertos señaló las siguientes ${ }^{14}$ : bar, acto social, vermú, niños en un bar, comida en un bar e ingesta de alcohol.

Como se desprende de los datos contenidos en la tabla que se muestra a continuación, no hay ningún porcentaje que sobresalga de una manera muy diferenciada del resto, si exceptuamos tal vez el de tapa, apuntada como problema cultural por el $27,02 \%$ en $1^{\circ}$ y por el $35,13 \%$ en $4^{\circ}$, y los nombres propios, como se puede observar en la tabla 12.

En la tabla 13 solo hemos dado ejemplos de aquellos tipos de problemas culturales que han alcanzado un porcentaje más llamativo de acuerdo con los resultados obtenidos:

Problemas pragmáticos. A continuación se recogen los problemas derivados del encargo de traducción, las características del receptor del texto, del contexto en el que se efectúa la traducción, así como con la función del texto (escopo) y/o con la intención del mismo. Los estudiantes que reconocen problemas de esta naturaleza en cada uno de los momentos formativos objeto de estudio quedan repartidos de la siguiente manera (ver tabla14):

\footnotetext{
14 Aquellos tipos que no se incluyen en las tablas de frecuencias se debe a que ninguno de los estudiantes participantes en la investigación reparó en estos aspectos apuntados inicialmente por el grupo de expertos.
} 
Tabla 12

Número y tipo de problemas culturales señalados por los estudiantes

Primer año

Presuposiciones señalado

no señalado
Cuarto año

señalado

no señalado

\begin{tabular}{|c|c|c|c|c|c|c|c|c|}
\hline & Frecuencia & $\%$ & Frecuencic & $\%$ & Frecuencia & $\%$ & Frecuencia & $\%$ \\
\hline tapa & 10 & $27,02 \%$ & 27 & $72,98 \%$ & 13 & $35,13 \%$ & 24 & $64,87 \%$ \\
\hline comida & 8 & $21,62 \%$ & 29 & $78,38 \%$ & 12 & $32,43 \%$ & 25 & $67,57 \%$ \\
\hline merienda & 2 & $5,4 \%$ & 35 & $94,6 \%$ & 2 & $5,4 \%$ & 35 & $94,6 \%$ \\
\hline vermú & 5 & $13,51 \%$ & 32 & $86,49 \%$ & 15 & $40,54 \%$ & 22 & $59,46 \%$ \\
\hline ingesta de alcohol & 2 & $5,4 \%$ & 35 & $94,6 \%$ & N/A & N/A & 37 & $100 \%$ \\
\hline nombres propios & 6 & $16,21 \%$ & 31 & $83,79 \%$ & 24 & $64,86 \%$ & 13 & $35,14 \%$ \\
\hline $\begin{array}{l}\text { variedad regional } \\
\text { nombres propios }\end{array}$ & $\mathrm{N} / \mathrm{A}$ & $\mathrm{N} / \mathrm{A}$ & 37 & $100 \%$ & 1 & $2,7 \%$ & 36 & $97,3 \%$ \\
\hline $\begin{array}{l}\text { relaciones } \\
\text { en el texto }\end{array}$ & 1 & $2,7 \%$ & 36 & $97,3 \%$ & 1 & $2,7 \%$ & 36 & $97,3 \%$ \\
\hline concepto tiempo & 1 & $2,7 \%$ & 36 & $97,3 \%$ & N/A & N/A & 37 & $100 \%$ \\
\hline $\begin{array}{l}\text { organización de la } \\
\text { semana (domingo) }\end{array}$ & 3 & $8,1 \%$ & 34 & $91,9 \%$ & 3 & $8,1 \%$ & 34 & $91,9 \%$ \\
\hline $\begin{array}{l}\text { horas de las } \\
\text { comidas (horarios) }\end{array}$ & 3 & $8,1 \%$ & 34 & $91,9 \%$ & 11 & $29,72 \%$ & 26 & $70,28 \%$ \\
\hline Total & 37. & & & $100 \%$ & 37 & & & $100 \%$ \\
\hline
\end{tabular}

\section{Tabla 13}

Ejemplos de los argumentos que apuntan problemas culturales

Ejemplos

tapa

variedad

regional

nombres

propios
10

$4^{0}$

\section{Tabla 14}

Número de estudiantes que reconocen problemas pragmáticos

\begin{tabular}{|l|c|c|c|c|}
\hline & Frecuencia & \% válido & Frecuencia & $\%$ válido \\
\hline Sí & 10 & & & 40 \\
\hline No & 14 & $37,83 \%$ & 22 & $59,45 \%$ \\
\hline total & 23 & $62,17 \%$ & 15 & $40,55 \%$ \\
\hline
\end{tabular}

Elemento cultural.

Es algo cultural.

Hay una gran carga cultural.

Hacen referencia a una tradición cultural (...) más si lo unimos a que esos nombres pertenecen a distintas lenguas dentro del territorio español. 
Los resultados obtenidos ponen de manifiesto que los estudiantes parecen experimentar una evolución de primero a cuarto en lo que respecta a los aspectos pragmáticos involucrados en el proceso traductor. Como puede apreciarse en la tabla 15, los estudiantes reconocen un mayor número de problemas de naturaleza pragmática en $4^{\circ}$ :

\section{Tabla 15}

Número y tipo de problemas pragmáticos señalados por los estudiantes

\begin{tabular}{|c|c|c|c|c|c|c|c|c|}
\hline & \multicolumn{4}{|c|}{ Primer año } & \multicolumn{4}{|c|}{ Cuarto año } \\
\hline & \multicolumn{2}{|c|}{ señalado } & \multicolumn{2}{|c|}{ no señalado } & \multicolumn{2}{|c|}{ señalado } & \multicolumn{2}{|c|}{ no señalado } \\
\hline & Frecuencia & $\%$ & Frecuencia & $\%$ & Frecuencia & $\%$ & Frecuencia & $\%$ \\
\hline encargo & N/A & N/A & 37 & $100 \%$ & 4 & $10,81 \%$ & 33 & $89,19 \%$ \\
\hline receptor & 14 & $37,83 \%$ & 23 & $62,17 \%$ & 15 & $40,54 \%$ & 22 & $59,46 \%$ \\
\hline formato & $\mathrm{N} / \mathrm{A}$ & N/A & 37 & $100 \%$ & 1 & $0,5 \%$ & 389 & $99,5 \%$ \\
\hline espacio & $\mathrm{N} / \mathrm{A}$ & N/A & 37 & $100 \%$ & 3 & $3,3 \%$ & 378 & $96,7 \%$ \\
\hline función & N/A & N/A & 37 & $100 \%$ & 2 & $5,4 \%$ & 35 & $94,6 \%$ \\
\hline intención & N/A & N/A & 37 & $100 \%$ & 5 & $1,3 \%$ & 386 & $98,7 \%$ \\
\hline Total & \multicolumn{4}{|c|}{ 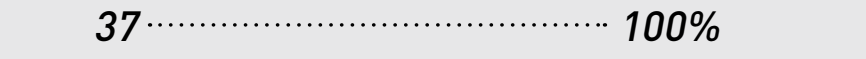 } & \multicolumn{2}{|l|}{37} & \multicolumn{2}{|c|}{$100 \%$} \\
\hline
\end{tabular}

En la tabla 16 se incluyen algunos ejemplos del metalenguaje utilizado a la hora de describir los problemas pragmáticos:

\section{Tabla 16}

Ejemplos de los argumentos que apuntan problemas pragmáticos

\begin{tabular}{|c|c|c|}
\hline Ejemplos & 10 & $4^{0}$ \\
\hline encargo & $\begin{array}{l}\text { Ningún estudiante en primero ha encontrado } \\
\text { problemas de este tipo. }\end{array}$ & $\begin{array}{l}\text { Ajustarse al encargo de traducción } \\
\text { Habría que ver el encargo. } \\
\text { Según el encargo. } \\
\text { Depende del encargo. }\end{array}$ \\
\hline receptor & $\begin{array}{l}\text { Para la comprensión del público extranjero. } \\
\text { Persona que lo va a leer. } \\
\text { Los ingleses. } \\
\text { Al no ser nuestra comunidad de hablantes. }\end{array}$ & $\begin{array}{l}\text { Los destinatarios. } \\
\text { El lector meta. } \\
\text { Los receptores. } \\
\text { Cualquier pasajero. } \\
\text { Un extranjero. }\end{array}$ \\
\hline
\end{tabular}




\section{DISCUSIÓN}

En total contamos con 37 estudiantes de la Universidad de Granada y sus respuestas en dos momentos de su formación: en la primera semana de acceso a los estudios de TI y en las últimas semanas próximas al egreso de la titulación en TI. El rango de edad modal de nuestra muestra se sitúa entre los 17 a los 20 años en el momento de ingreso y, lógicamente, cuatro años más tarde al tratarse de los mismos estudiantes, la edad modal es la que comprende entre los 21 y 24 años. En lo que se refiere al sexo de la muestra, contamos con una amplia mayoría de mujeres (27) y 10 hombres. En cuanto a la distribución por sexos de los estudiantes de TI en España, y siempre de acuerdo con los datos obtenidos en nuestro macro-estudio (Gregorio Cano, 2014), queda patente que la mayoría son mujeres, hecho que coincide con la tradición en la rama de Humanidades y, en particular, en las titulaciones relacionadas con idiomas (Filologías, Estudios de Lenguas y Culturas, entre otros), como recogen los informes del Ministerio de Ciencia e Innovación $(2008,2010)$, asimismo en sintonía con los datos obtenidos por Calvo (2010, p. 383), así como con el estatus de pink collar que otorga Katan (2011, p. 149) a la disciplina de TI.

En el caso de la UGR, la oferta de lenguas B es la más amplia de España con 4 lenguas B, a saber: alemán, árabe, francés e inglés. Contamos con estudiantes de tres de las cuatro lenguas, ningún estudiante de árabe cumplimentó la herramienta en los dos momentos objeto de estudio de nuestra investigación. Por otro lado, ninguno de los participantes cuenta con estudios universitarios previos al ingreso en TI.

Gracias al estudio de casos intensivo podemos concluir que no existe ningún rasgo común para los estudiantes que evolucionan, salvo las estancias en algún país de la lengua B. Asimismo, con los datos obtenidos no se puede determinar qué factor (o factores) resulta determinante en un mayor o menor conocimiento y uso del metalenguaje, a partir de la cumplimentación de nuestra herramienta.

Somos conscientes de que los resultados presentados en este artículo cuentan con ciertas limitaciones, pero creemos que los datos extraídos de las respuestas de un mismo grupo de estudiantes en dos momentos de su formación puede parecer que nos conducen a algunas conclusiones lógicas, como que el mayor o menor desarrollo en el uso del metalenguaje depende de características individuales, ya que con los datos disponibles no podemos establecer patrones comunes a los 37 participantes en el estudio longitudinal.

Los datos que hemos presentado se corresponden con una muestra de 37 estudiantes, por lo que no pretenden ser extrapolables a toda la población, sino servir como ejemplo empírico de la evolución (o no) que experimentan los estudiantes de TI de un mismo centro a la hora de identificar problemas de traducción y hablar de su campo de conocimiento tras cuatro años de formación.

El uso del metalenguaje para describir los distintos problemas presentes en el texto en $1^{\circ}$ y en $4^{\circ}$ resulta ser un parámetro en el que se registra una evolución como se ha podido constatar tras el análisis del estudio caso por caso, donde 22 de los 37 demuestran un dominio mayor. Asimismo, una de las primeras conclusiones a las que llegamos es que no existen diferencias sustanciales a la hora de reconocer un determinado tipo de problema de traducción en $1^{\circ}$ y en $4^{\circ}$, el hecho de que se identifiquen más o menos problemas en $1^{\circ}$ o en $4^{\circ}$ parece deberse a características individuales, como se expresa en el macro-estudio (Gregorio Cano, 2014). 
En cuanto a los problemas lingüísticos, a lo largo de nuestra investigación se constata que existe cierta tendencia a la palabra entre los estudiantes en ambos momentos formativos, aunque sí existen diferencias a la hora de describirlos. Por otro lado, los problemas textuales no centran especialmente la atención de ninguno de los grupos poblacionales, aunque sí que los estudiantes de $4^{\circ}$ demuestran una mayor toma de conciencia respecto a la presencia de estos problemas en el encargo incluido en el estudio. El texto incluido en el encargo de pre-traducción estaba plagado de elementos culturales, que han sido reconocidos casi unánimemente por ambos grupos poblacionales, aunque la manera de referirse a ellos sí registra una evolución de $1^{\circ}$ a $4^{\circ}$. Por último, los problemas de naturaleza pragmática también registran una evolución tanto a la hora de identificar un mayor número en $4^{\circ}$ que en $1^{\circ}$, así como a la hora de describirlos.

Sin embargo, no se reconoce ningún patrón que guíe el desarrollo de la capacidad de identificación y descripción de problemas. Por último, a pesar de que no existen diferencias sustanciales entre la capacidad a la hora de reconocer un determinado tipo de problema en $1^{\circ}$ y en $4^{\circ}$, se constata a partir de los ejemplos incluidos en este artículo que la principal diferencia (evolución) radica en la manera de describir los problemas, es decir, el metalenguaje sí experimenta una evolución al denotar una mayor toma de conciencia de los factores involucrados en el proceso de traductor a partir de las palabras utilizadas por los estudiantes de traducción.

Uno de los resultados más enriquecedores de este tipo de estudios es la gran cantidad de ideas que han surgido durante y como fruto del proceso investigador. La experiencia nos ha hecho ver algunas mejoras posibles hacia un futuro estudio longitudinal de similares características con el fin de pulir ciertos puntos débiles o a los que no hemos podido dar respuesta por ciertas carencias de información. En este sentido, a la hora de planificar nuestro próximo estudio longitudinal queremos contar con dos grupos (uno de ellos que sea nuestro grupo control). Asimismo, diseñaremos un estudio longitudinal reducido en el que los datos de nuestra herramienta se verán completados con la traducción de la tarea y entrevistas retrospectivas con los estudiantes participantes con el fin de profundizar en el conocimiento de nuestro ámbito de estudio (la competencia traductora), así como con una serie de encuentros con los profesores responsables de las asignaturas cursadas por los participantes. De este modo, podremos identificar posibles tendencias que indiquen factores comunes o diferentes que influyen en la capacidad para detectar los problemas de traducción lestilos de aprendizaje, enfoques docentes, por ejemplo), como parte del desarrollo de la competencia traductora. 


\section{REFERENCIAS}

Buendía, L. \& Colás, P. (1998). Investigación Educativa. Sevilla: Alfar.

Buendía, L., Colás, Ma P. \& Hernández, F. (1998). Métodos de investigación en psicopedagogía. Madrid: McGraw-Hill.

Calvo Encinas, E. (2010). Análisis curricular de los estudios de Traducción e Interpretación en España: perspectiva del estudiantado (Tesis doctoral inédita). Granada: Universidad de Granada.

Elena, P. (2011). Bases para la comprensión organizativa del texto. Revista de Lingüística y Lenguas Aplicadas, 6, 125-137.

Delisle, J., Lee-Jahnke, H. \& Cormier, M. (Eds.) (1999). Terminologie de la traduction/Translation terminology/ Terminología de la traducción/ Terminologie der Übersetzung. Ámsterdam y Filadelfia: John Benjamins.

Fink, A. (2003). The Survey Handbook. Thousand Oaks: SAGE.

Gambier, Y. \& Van Doorslaer, L. (2009). The Metalanguage of Translation (Vol. 20). Ámsterdam y Filadelfia: John Benjamins.

García de Toro, C. \& García Izquierdo, I. (2005). La práctica profesional de la traducción. En C. García de Toro \& I. García Izquierdo (Eds.), Experiencias de traducción: Reflexiones desde la práctica traductora (pp. 15-30). Castellón: Universitat Jaume I.

García Ferrando, M. (2005). La encuesta. En F. Alvira, J. Ibáñez \& M. García (Eds.), El análisis de la realidad social. Métodos y técnicas de investigación (pp. 167-201). Madrid: Alianza Editorial.

García Izquierdo, I. (2000). Análisis textual aplicado a la traducción. Valencia: Tirant lo Blanch.

García Izquierdo, I. (2012). Competencia textual para la traducción. Valencia: Tirant Humanidades.

Gile, D. (2009). Basic Concepts and Models for Interpreter and Translator Training. Ámsterdam y Filadelphia: John Benjamins.

Gregorio Cano, A. (2014). Estudio Empírico-descriptivo del Desarrollo de la Competencia Estratégica en la Formación de Traductores (Tesis doctoral inédita). Granada: Universidad de Granada.

Gregorio Cano, A. (en prensa). El perfil de ingreso y egreso de los estudiantes de Traducción e Interpretación como elemento clave en el desarrollo de la competencia traductora. Hermeneus.

Hurtado Albir, A. (2011). Traducción y Traductología. Introducción a la Traductología. Madrid: Cátedra.

Hurtado Albir, A. (2017) (Ed.). Researching Translation Competence by PACTE Group. Ámsterdam: John Benjamins.

Katan, D. (2011). Occupation or Profession: A Survey of the Translators' World. En R. Sela-Sheffy \& M. Shlesinger (Eds.), Profession, Identity and Status: Translators and Interpreters as an Occupational Group (pp. 187-209). Filadelfia: John Benjamins.

Kelly, D. (2002). Un modelo de competencia traductora: bases para el diseño curricular. Puentes. Hacia Nuevas Investigaciones en la Mediación Intercultural, 1, 9-20.

Kelly, D. (2005). A Handbook for Translator Trainers. A Guide to Reflective Practice. Mánchester: St. Jerome.

La Rocca, M. (2007). El taller de traducción: una metodología didáctica integradora para la enseñanza universitaria de la traducción (Tesis doctoral inédita). Vic: Universitat de Vic.

Mayoral, R. (1999). La traducción de referencias culturales. Sendebar, 12, 67-88.

Mayoral, R. (2001). Aspectos epistemológicos de la Traducción. Castellón de la Plana: Publicaciones de la Universitat Jaume I.

Mayoral, R. \& Díaz Fouces, Ó. (2011). Sobre las especialidades de la traducción y la traducción especializada. Castellón de la Plana: Universidad Jaume I.

Ministerio de Ciencia e Innovación (2008). Estudio de la oferta, la demanda y la matrícula de nuevo ingreso en las Universidades públicas y privadas para el curso 2007/08. Recuperado de https://goo.gl/3FHM6q

Ministerio de Ciencia e Innovación (2010). Estudio de la oferta, la demanda y la matrícula de nuevo ingreso en las Universidades públicas y privadas para el curso 2009/10. Recuperado de https://goo.gl/2xZAii

Neubert, A. \& Shreve, G. (1992). Translation as Text. Kent: The Kent State U.P.

Nord, C. (1991). Text Analysis in Translation. Theory, Methodology and Didactic Application of a Model for Translation-Oriented Text Analysis. Ámsterdam: Rodopi.

Nord, C. (1997). Translating as a Purposeful Activity, Functionalist Approaches Explained. Mánchester: St. Jerome.

Orozco, M. (2000). Instrumentos de medida de la adquisición de la competencia traductora: construcción y validación (Tesis doctoral inédita). Barcelona: Universitat Autònoma de Barcelona.

PACTE. (2011a). Results of the Validation of the PACTE Translation Competence Model: Translation Problems and Translation Competence. En C. Alvstad, A. Hild \& E. Tiselius (Eds.), Methods and Strategies of Process Research: Integrative Approaches in Translation Studies (pp. 317-343). Ámsterdam y Filadelfia: John Benjamins. 
PACTE. (2011b). Results of the validation of the PACTE translation competence model: Translation project and dynamic translation index. En S. O'Brien (Ed.), Cognitive Explorations of Translation (pp. 30-53). Londres/Nueva York: Continuum Studies in Translation.

Presas, M. (1996). Problemes de traducció i competencia traductora (Tesis doctoral inédita). Barcelona: Universitat Autònoma de Barcelona.

Rialp, Á., Martínez, P. \& Rialp, J. (2005). El desarrollo exportador de las pymes industriales españolas participantes en un consorcio de exportación: un estudio de caso. Cuadernos de Gestión, 5(2), 95-116.

Roiss, S. (2006a). El pacto de lectura y la adquisición de la competencia traductora: un ejemplo de traducción especializada inversa lespañol-alemán). TRANS, 10, 99-112.

Roiss, S. (2006b). Competencia traductora y tipología textual: En busca del punto de intersección. Estudios Filológicos Alemanes, $11,439-450$.

Rojas, A. J., Fernández, J. A. \& Pérez, C. (Eds.) (1998). Investigar mediante encuestas. Fundamentos teóricos y aspectos prácticos. Madrid: Síntesis.

Shreve, G. (1997). Cognition and the evolution of translation competence. En J. Danks, G. Shreve, S. Fountain y M. McBeath (Eds.), Cognitive Processes in Translation and Interpreting (pp. 120-136). Thousand Oaks: Sage Publications.

Sierra, R. (1998). Técnicas de investigación social. Teoría y ejercicios. Madrid: Paraninfo.

Tang, J. (2007). The metalanguage of translation. A Chinese perspective. Target, 19(2), 359-374.

(c) Los autores. Este artículo es publicado por la Revista Digital de Investigación en Docencia Universitaria del Área de Institutional Research and Effectiveness de la Dirección de Aseguramiento de la Calidad, Universidad Peruana de Ciencias Aplicadas. Este es un artículo de acceso abierto, distribuido bajo los términos de la LicenciaCreativeCommons Atribución-Compartirlgual 4.0 Internacional.l http://creativecommons.org/licenses/bysa/4.0/l, que permite el uso no comercial, distribución y reproducción en cualquier medio, siempre que la obra original sea debidamente citada. 